local/territorial qui depuis qu'ils ont quitté les bancs de l'université ne trouvent pas le temps d'approfondir leurs connaissances par la lecture de savants traités sous la forme de «brique ». Cet ouvrage saura répondre à leurs désirs de se familiariser avec certaines idées ou certains concepts sans devoir y investir trop de temps et d'argent.

$* * *$

\section{Rodrigue Tremblay, Le code pour une éthique globale, Montréal, Liber, 2009, 287 p.}

Voici un ouvrage qui pourra surprendre les lecteurs de O\&T, car il n'est pas question du moins de façon explicite - de territoires. Quant à ce qui se rapporte à l'organisation, c'est de celle de la société dans son ensemble qu'il est question, par la référence à dix règles humanistes sur lesquelles elle devrait reposer afin de s'assurer d'affronter avec succès les défis auxquels elle doit faire face. Rodrigue Tremblay, détenteur d'un Ph. D. de Stanford University, est professeur émérite d'économie de l'Université de Montréal. Les gens de ma génération se rappelleront des années où il fut dans le premier cabinet de René Lévesque ministre de l'Industrie et du Commerce. Auteur prolifique, mes propres étudiants ont pu bénéficier de ses ouvrages de micro et de macroéconomie. Par ce volume d'un genre tout à fait différent, l'auteur ambitionne ici de montrer qu'entre l'absence totale de morale et d'éthique et des normes morales religieuses inadéquates, la seule voie morale qui s'ouvre aux citoyens du monde est humaniste, rationnelle et universelle.

En préface, Paul Kuritz, président fondateur du Council for Secular Humanism considère que face aux problèmes contemporains qui menacent l'humanité, il existe un scénario de nature optimiste. C'est le scénario humaniste qui postule qu'en mettant l'accent sur la raison, la science et la technologie en tant que facteurs essentiels pour améliorer la condition humaine, les êtres humains sont en mesure d'affronter les défis énormes qui se présentent à eux en ce $\mathrm{XXI}^{\mathrm{e}}$ siècle. Mais, pour ce faire, le monde aurait besoin d'un nouveau siècle des Lumières qui réaffirmerait la primauté de la raison, de la science, de l'éducation et de la démocratie. C'est ce que cherche à démontrer Rodrigue Tremblay en puisant dans son immense culture. L'humanisme se trouve défini ici comme une vision philosophique de l'humanité et de l'existence humaine qui proclame la dignité inhérente de tous les êtres humains et leur valeur intrinsèque, sans référence aucune aux superstitions et à la notion de surnaturel. L'humanisme rationnel, comme il est signalé, fait appel à la logique et à la science pour comprendre l'univers et pour résoudre les problèmes de l'existence, en invitant chacun à vivre une vie morale de la meilleure des façons possibles.

Chacun des dix chapitres du volume se trouve lié à une règle propre à l'humanisme. Ainsi, le deuxième chapitre Respect se réfère à la règle qui proclame le respect de la vie et de la propriété d'autrui en tout temps. Les extrémistes de gauche comme de droite sont invités à s'abstenir. On y trouve les cinq principales catégories de lacune des marchés, lesquelles se rapportent aux biens publics, aux monopoles, aux externalités négatives, aux informations incomplètes et aux disparités excessives dans la répartition de la richesse. On l'aura compris, ici l'État a un rôle à jouer, l'auteur étant toujours à ce qu'il me semble, d'obédience keynésienne. Mais il ne manque pas de mettre en garde contre des interventions étatiques susceptibles de susciter le choléra en voulant combattre la peste. C'est pourquoi Tremblay recommande la mise en branle d'une réforme du rôle régulateur de l'État avant d'entreprendre une réglementation en profondeur des marchés privés. 
Le chapitre 3, Tolérance, ne manque pas d'intéresser le lecteur québécois qui a encore à l'esprit les débats soulevés par la question des accommodements raisonnables et la commission Bouchard-Taylor qui s'en est suivie. Le principe humaniste ici concerné est la tolérance et l'ouverture d'esprit face aux choix et aux modes de vie des autres. Mon collègue de l'Université de Montréal estime que l'une des plus grandes menace contre la liberté et la démocratie de nos jours nous vient du multiculturalisme qu'il écrit comme une doctrine fallacieuse (merci P.E. Trudeau!) ayant comme principe sous-jacent que toutes les cultures sont égales. En conséquence, selon cette idéologie, est-il signalé, il faudrait préserver l'identité culturelle de groupes ethniques qui immigrent dans une société donnée même lorsque leurs valeurs culturelles sont fondamentalement hostiles à la liberté et à la démocratie. C'est ainsi que l'on peut voir depuis quelques années des burqas et autres niquabs au marché Jean-Talon de Montréal ${ }^{1}$. Donc, pour l'auteur, s'il faut être tolérant, il ne faut pas l'être façon bêtement naïve.

Il m'apparaît que le chapitre 7, Environnement, est celui qui intéressera le plus les lecteurs d'O\&T. La règle humaniste ici prise en considération s'applique à conserver et améliorer l'environnement naturel de la terre en tant qu'héritage commun de l'humanité. L'érudition de l'auteur, qui est marquante à l'intérieur de tous les chapitres, impressionnera ceux qui, comme l'auteur de ces lignes, se passionnent pour l'astrophysique et la paléoanthropologie. Mon collègue se demande si les humains, à l'instar des dinosaures, ne sont pas appelés à disparaître de la surface de la planète étant donné à la fois les torts causés à l'environnement et le pouvoir dévastateur des armes de destruction massive. L'homme n'a pas le choix : il s'adapte ou il disparaît. L'adaptation, on le sait, se veut la première loi de survie des organismes vivants. Le chapitre 9, Démocratie, s'avère tout aussi intéressant. La règle humaniste correspondante vise l'organisation de l'espace public selon les principes de liberté et de responsabilité en s'appuyant sur la démocratie politique et économique. Cinq dangers guettant la démocratie sont ici mis en évidence : un trop grand écart entre les riches et les pauvres; l'endettement, le sous-financement public et l'inflation; la prise du pouvoir par des sociopathes ou autres psychopathes; la malédiction de la propagande étatique (on passe du nazisme à la Maison Blanche occupée par G.W.Bush); la concentration de la propriété des organes de presses et le financement des campagnes électorales. Ce dernier danger est bien familier aux Québécois, hélas. Ces mises en garde étant faites, on ne peut qu'être d'accord avec Rodrigue Tremblay quand il soutient que le développement de toute économie repose sur la capacité d'innover. L'innovation en tant que levain de la croissance économique est tributaire de la liberté de penser et d'agir au sein de la société.

Le lecteur ne pourra qu'apprécier l'abondante documentation en langue française et anglaise sur laquelle l'argumentation de l'auteur prend son appui. Le style alerte et efficace, à défaut de convaincre tous les lecteurs sans exception, aura à tout le moins pour conséquence d'inviter à réfléchir sur les réalités qui entourent l'avenir de l'humanité.

${ }^{1}$ C'est moi qui le signale.

$* * *$

\section{Benoit Meyronin, Le marketing Territorial : enjeux et pratiques, Paris, Vuibert, 2009, 259 p.}

Le marketing, tout le monde connaît, mais appliqué à la problématique territoriale, il s'agit d'un usage assez récent, car l'expression n'a fait son apparition seulement il y a 